\title{
Prosthetic rehabilitation with dental implants after treatment of a keratocystic odontogenic tumor. case report
}

\author{
Amila Brkić ${ }^{1}$, Hülya Koçak-Berberoğlu ${ }^{2}$, Esra Eyupoğlu ${ }^{2}$, Vakur Olgaç ${ }^{3}$ \\ ${ }^{1} \mathrm{DDS}, \mathrm{PhD}$. Department of Oral Surgery, Sarajevo University School of Dentistry, Sarajevo, Bosnia and Herzegovinia \\ ${ }^{2} \mathrm{DDS}, \mathrm{PhD}$. Department of Oral and Maxillofacial Surgery, Istanbul University School of Dentistry, Istanbul, Turkey \\ ${ }^{3} \mathrm{DDS}, \mathrm{PhD}$. Institute of Oncology, Medical Faculty, Istanbul University Çapa, Istanbul, Turkey
}

Correspondence:

Sarajevo University, School of Dentistry

Department of Oral Surgery

71000 Sarajevo, Bosnia and Herzegovina

Tel: 0038762268134

E-mail:amilabrkic@hotmail.com

Brkić A, Koçak-Berberoğlu H, Eyupoğlu E, Olgaç V. Prosthetic rehabilitation with dental implants after treatment of a keratocystic odontogenic tumor. case report. J Clin Exp Dent. 2011;3(Suppl1):e360-4.

http://www.medicinaoral.com/odo/volumenes/v3iSuppl1/jcedv3iSuppl1p360.pdf

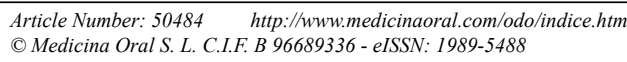

eMail: jced@jced.es

\begin{abstract}
Keratocystic odontogenic tumor (KCOT), formerly known as the odontogenic keratocyst (OKC) is a benign intraosseous lesion, characterized by invasive growth and high rate of recurrences. Although it is not frequently seen, KCOT may be associated with unerupted teeth and without relevant histopathological examinations, might be mistaken with dentigerous cyst.

A 26 year-old male patient referred to our department with complaints of two months persisting painless swelling on the left side of mandible. Clinical and radiographic examinations revealed an impaction of the horizontally positioned left lower second premolar surrounded by a well-circumscribed radiolucent mass, located between the left canine and the second molar, suspected to be a cyst.

The aim of this report is to present a therapeutical approach to keratocystic odontogenic tumor associated with impacted tooth and prosthetic rehabilitation with dental implants after an initial treatment. The patient is under follow up for 31 months since implant placement was done, without any complaints or complications.
\end{abstract}

Key words: keratocystic odontogenic tumor, drainage, impaction, dental implants. 


\section{Introduction}

The odontogenic keratocyst (OKC), now officially known as the keratocystic odontogenic tumor (KCOT), is a benign jaw lesion, characterized by invasive growth and high rate of recurrences (1-3). Etiology of its occurrence include dental lamina remnants and extension of basal cells of overlying oral epithelium $(1,2)$. However, by some authors DNA mutations might be also included as the etiological factors, which is supported by malignant transformation of the cyst with a frequency rate from 5 to $62,5 \%$ (1-3). By these evidences, developmental theory of OKC occurrence might be discarded, and changed to neoplastic theory (2). Due of neoplastic features, in 2005 the World Health Organization have made a new classification of head and neck tumors, in which "odontogenic keratocyst" has been renamed to "keratocystic odontogenic tumor"(KCOT) (1). Clinically as the unilocular or multilocular jaw lesions, KCOTs are mostly seen in male patients, in their second and third decades (1-3). Occurrence of the multilocular lesions in child patients, might be associated with Gorlin-Goltz (nevoid basal cell carcinoma) syndrome $(2,3)$. In a $3 / 4$ of all cases, the most common site for keratocystic odontogenic tumor is mandibular molar and ramus region $(1,2)$. A localized asymptomatic swelling associate with mobility of the teeth, and spontaneous drainage of the tumour into the oral cavity, are the most common symptomes of the KCOT occurrence $(1,3)$. Radiographically, the tumor is characterized as well borded radiolu- cent lesion with smooth and usually sclerotic margins (3). One of the important characteristics of KCOTs is their association with impacted teeth, with occurrence rate from 25 to $40 \%$, which should not be neglected, due to differential diagnosis to dentigerous cysts (1-3). Also, it is reported that KCOTs in 10 to $15 \%$ of cases erode the adjacent roots $(1,2)$. Histologically, KCOTs are characterized by a thin squamous epithelium covered by keratin, according which, they are subdivided into parakeratotic and orthokeratotic subtypes $(1,2)$. Parakeratotic is more frequently seen than orthokeratotic subtype, and is characterized by aggressive behavior, which include high rate of recurrences (1). Basal layer of the tumor epithelium might be budding into a supporting connective tissue, forming so called daughter cysts, which are mostly responsible for tumour reccurrences (2).

The aim of this report is to present a case of keratocystic odontogenic tumor associated with impacted tooth and prosthetic rehabilitation with dental implants after an initial treatment.

\section{Case Report}

In March 2006, a 26-year-old male patient was referred to department of Oral surgery, Faculty of Dentistry University of Istanbul with a complaint of painless swelling in the region of left mandible, which persisted for two months. Clinical examinations revealed a soft expansion in the region between the lower left canine and the first molar, from the vestibular side of the mandible. The sur-

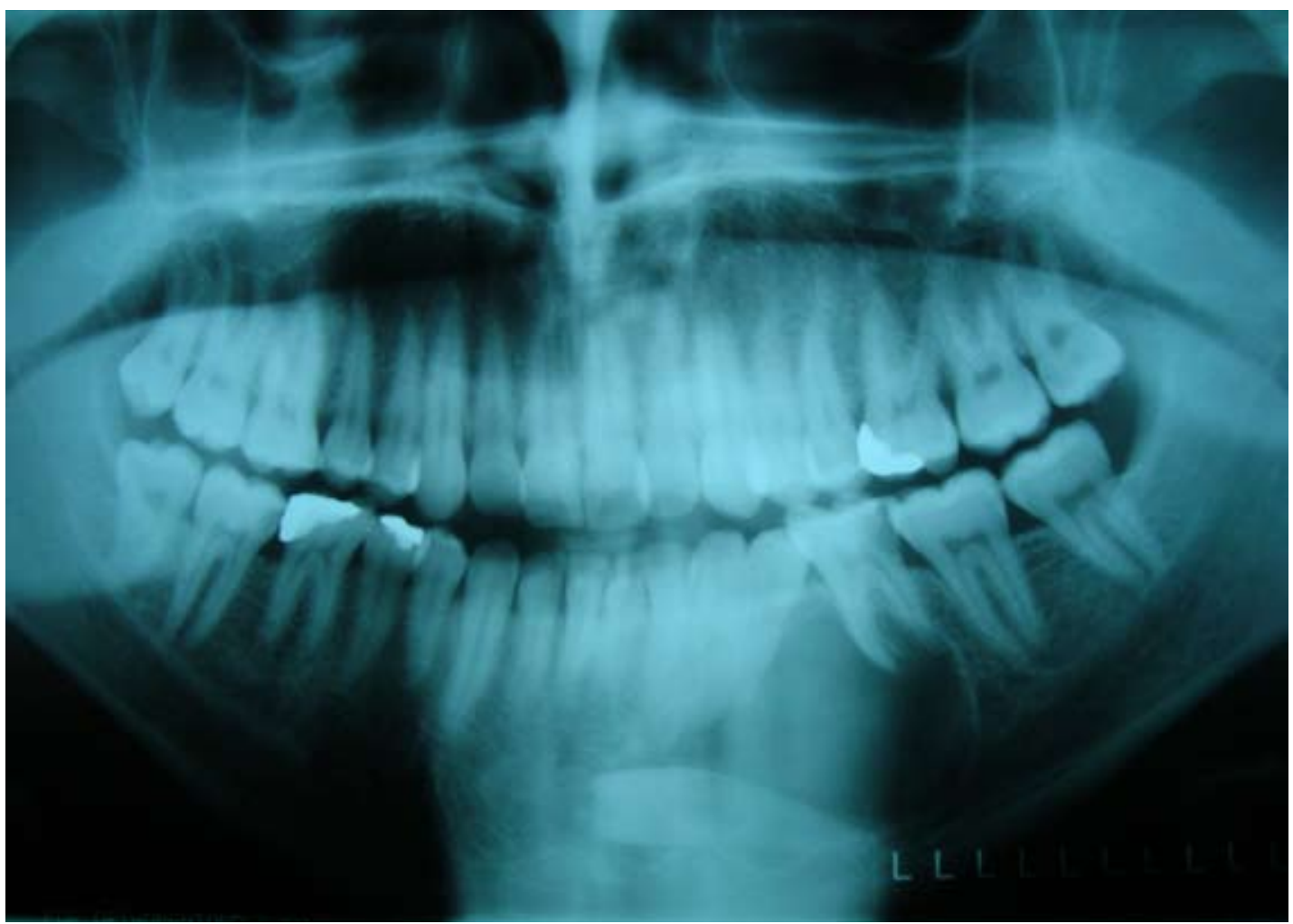

Fig. 1. Preoperative panoramic radiograph shows impaction of left lower second premolar surrounded by "cystic" formation. 
face was smooth and the colour was consistent with the adjacent mucosa. The left lower second premolar was missing, while the first premolar and the first molar were mobile. There were no symptoms of pain or paresthesia. Extraorally, no presence of asymmetry or lymphadenophaty was noted. The panoramic radiographs disclosed the impaction of the horizontally positioned left lower second premolar surrounded by a well-circumscribed radiolucent mass located between the left canine and the second molar, suspected to be a cyst. The lower edge of the pathological formation was in the level of the inferior border of the mandible (Fig. 1). Under local anesthesia, the first premolar tooth was extracted, and a polyethylene drainage tube was placed in the socket, facilitating decompression of the cyst (Fig. 2). Biopsy of the lesion was done and result of histopathological evaluation showed a parakeratotic type of keratocystic odontogenic tumor.

During the six-months decompression period, every 7 th day a debridement of the drainage tube with irrigation of tumor cavity by isotonic solution were performed. After the decompression, enucleation and surgical extraction of the impacted tooth were done. The left canine and the first molar were extracted due to their mobility.

The mass of the soft tissue enucleated from the area of impaction was submitted for histopathological evaluation. The examinations revealed a prominent granular cell layer with lamellar keratinization lying on fibrous connective tissue, which was diagnosed as the orthokeratinized odontogenic keratocyst. 17 months after the follow-up period, during which there were no signs of complications, such as the recurrence of the tumor or bad bone healing, the patient underwent implant surgery under local anesthesia following the routine oral implant protocol. Two ITI implants were placed at the former element sides of the canine $(3.3 \mathrm{~mm}, 12 \mathrm{~mm})$ and the first molar tooth $(3.3 \mathrm{~mm}, 10 \mathrm{~mm})$. During this surgery there was no need for bone augmentation, due a good bone preservation. After a 5-month healing period, in April 2008, the prosthetic rehabilitation was completed with the implantation of a porcelain bridge (Fig.3). During the following 31 months, no complications developed.

\section{Discussion}

One of the most important characteristics of keratocystic odontogenic tumor is a high rate of recurrence that ranges from $5-62 \%$, and is more higher for those tumors associated with Gorlin-Goltz syndrome (1-3). The level of the recurrence depends of therapeutic approaches, location of the tumor and post-operative follow-up period. Treatment options for KCOT include: simple curettage, enucleation (in combination with cryotherapy or Carnoy`s solution), marsupialization, decompression and secondary enucleation, and resection (marginal or segmental) (1,3-8). Enucleation is the treatment option which lead statistically the biggest number of recurrences, while in the case of resection, due its radical nature, recurrences are not noted $(1,3,7)$. Decompression is the modification of marsupialization characterized by relieving the intracystic pressure, which lead to reduction of the cyst size and new bone formation $(5,7)$. Marsupialization and decompression are the most common thera-

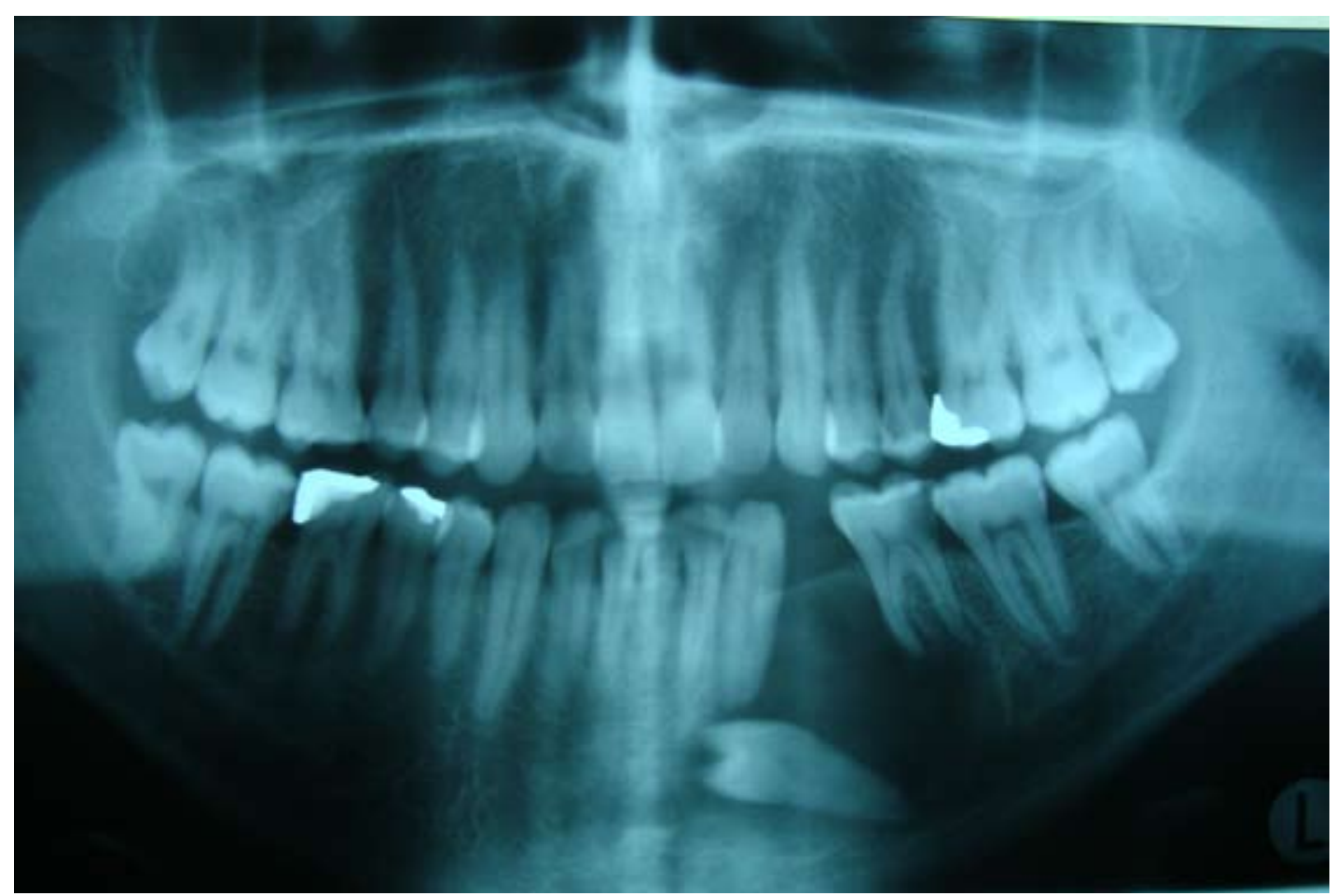

Fig. 2. Controle panoramic radiograph made during a tumor drainage-3th month. 


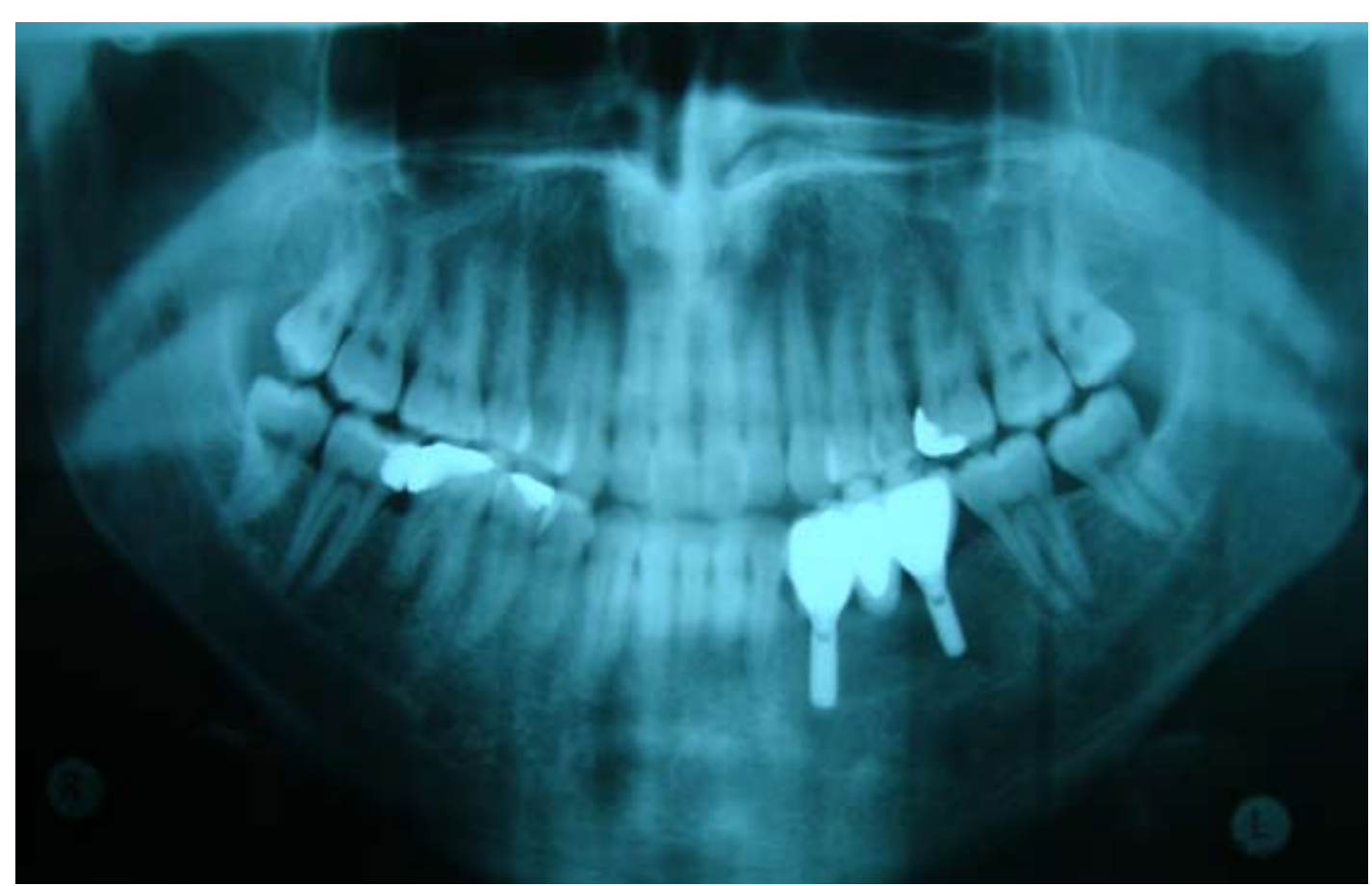

Fig. 3. Controle panoramic radiograph made 12 months after implant placement.

peutical procedures applied for treatment of large keratocystic odontogenic tumours, because they can save the anatomic structures including adjacent teeth, maxillary sinus or inferior alveolar nerve $(5,7,8)$. Also, different clinical studies have shown the another advantages of marsupialization and decompression in a treatment of large keratocystic odontogenic tumors $(5,7,8)$. Histopathological changes of the KCOTs epithelium after decompression, from aggressive parakeratized to less aggressive ortokeratized, even nonkeratinizing squamous epithelium, were reported by Nakamura et al (5), Jung et al. (7) and Brøndum and Jensen (8). Nakamura et al.(5) have also concluded that decompression is more effective in mandibular body than ramus region, while a bigger incidence of recurrences is noted in cases of multilocular lesions and the lesions located in the ramus region. In our presented case the tumor's histopathological changes were noted too; an evaluation of enucleated specimen after decompression was orthokeratinized odontogenic keratocyst, comparing with biopsy sample taken before the treatment. Brøndum and Jensen (8) in a case series of 12 patients who underwent the tumor drainage with polyethylene drainage tube, without presence of recurrences during follow-up period from 7 to 17 years, concluded that the decompression treatment reduce the tendency to recurrence of the odontogenic keratocyst. In our presented case, decompression and secondary enucleation of the keratocystic odontogenic tumor associated with impacted left lower second premolar, were performed.

Similar to cases of Brøndum and Jensen (8), we used polyethylene drainage tube implanted into a tumor cavity facilitating decompression of the tumor during a six months period. Radiographically a decreasing of tumor size with change of tooth inclination were noted. By Jung et al. (7), decompression can induce an eruption of impacted tooth associated with cyst reduction. However, in our case spontaneous eruption was not expected, due to the inclination of the impacted tooth, while the eruption by orthodontic tractions would present one kind of risk, following the aggressive behavior of the lesion.

In our report we presented also a prosthetic reconstruction with dental implants, after an initial therapy. Prosthetic rehabilitation with dental implants is the best solution for replacement of missing teeth in the cases of good bone preservation. In our case, 17 months after the follow-up period, the patient underwent implant surgery. Presented cases of treated jaw cysts and later implant placements by other authors have also showed a good results $(4,6)$.

In described cases of Barry and Kearns (4) and Karamanis et al. (6) implant placements were associated by bone grafting with corticocancellous iliac crest bone and alloplastic bone substitute graft. In our case there was no need for bone augmentation, due a good bone preservation and the patient is under follow up for 31 months since implant placement was done, without any complaints or complications.

However, a long-term follow-up is needed in order to avoid any unnecessary complications, such as implant failure or recurrence of the tumor, which might be noted even ten years after the treatment (1). 


\section{References}

1. Çakur B, Miloğlu Ö, Yolcu Ü, Göregen M, Gürsan N. Keratocystic odontogenic tumor invading the right maxillary sinus : a case report. J Oral Sci 2008; 50: 345-9.

2. Agaram NP, Collins BM, Barnes L, Lomago D, Aldeeb D, Swalsky $\mathrm{P}$ et al. Molecular analysis to demonstrate that odontogenic keratocysts are neoplastic. Arch Pathol Lab Med. 2004; 128:313-7.

3. Grasmuck EA, Nelson BL. Keratocystic odontogenic tumor. Head Neck Pathol. 2010; 4:94-6.

4. Barry CP, Kearns GJ. Case report--odontogenic keratocysts: enucleation, bone grafting and implant placement: an early return to function. J Ir Dent Assoc. 2003;49:83-8.

5. Nakamura N, Mitsuyasu T, Mitsuyasu Y, Taketomi T, Higuchi Y, Ohishi M. Marsupialization for odontogenic keratocysts: longterm follow-up analysis of the effects and changes in growth characteristics. Oral Surg Oral Med Oral Pathol Oral Radiol Endod. 2002; 94:543-53.

6. Karamanis S, Kitharas T, Tsoukalas D, Parissis N. Implant placement after marsupialization of a dentigerous cyst. J Oral Implantol. 2006; 32:313-6.

7. Jung YS, Lee SH, Park HS. Decompression of large odontogenic keratocysts of the mandible. J Oral Maxillofac Surg. 2005; 63:26771.

8. Brøndum N, Jensen VJ. Recurrence of keratocysts and decompression treatment. A long-term follow-up of forty-four cases. Oral Surg Oral Med Oral Pathol. 1991;72:265-9. 